\title{
Fluctuations of the Entropy Production in Anharmonic Chains
}

\author{
Luc Rey-Bellet[], Lawrence E. Thomas] \\ Department of Mathematics, University of Virginia \\ Kerchof Hall, Charlottesville VA 22903, USA
}

\begin{abstract}
We prove the Gallavotti-Cohen fluctuation theorem for a model of heat conduction through a chain of anharmonic oscillators coupled to two Hamiltonian reservoirs at different temperatures.
\end{abstract}

\section{Introduction}

The Gallavotti-Cohen fluctuation theorem refers to a symmetry in the fluctuations of the entropy production in nonequilibrium statistical mechanics. It was first discovered in numerical experiments of Evans, Cohen and Morris [8] and then discussed in [9] in the context of thermostated systems. As a mathematical theorem it was proved for Anosov dynamical systems [9, 10]. Soon thereafter the fluctuation theorem was discussed in the context of stochastic dynamical systems first by Kurchan [17] and then, more systematically by Lebowitz and Spohn, and Maes 22. 18. In particular, Maes discovered a general formulation of the fluctuation theorem in the context of space-time Gibbs measures which covers both Markovian stochastic dynamics and chaotic deterministic dynamics (via a Markov partition). As a mathematical theorem the fluctuation theorem is proven for quite general stochastic models with finite state space, such as lattices gases in a finite box. Relations for the free energy related to the fluctuation theorem have been also discussed in 15, 2.

Among the consequences of the fluctuation theorem is the non-negativity of entropy production although the proof of its positivity is more difficult and is so far proved only in particular examples [7, 20]. We also note that in the related context of open systems, classical and quantum, the production of entropy is discussed at a general level in 27, 13, 24. Again the non-negativity of entropy production is relatively easy to establish, while the strict positivity has been established only in particular models [7, 14].

In this paper we consider an open system consisting of a finite (but of arbitrary size) chain of anharmonic oscillators coupled at its ends only to reservoirs of free

\footnotetext{
${ }^{1}$ Email: lr7q@virginia.edu.

${ }^{2}$ Email: let@virginia.edu. Supported in part by NSF Grant 980139
} 
phonons at positive and different temperatures [6, 7, 5, 25, 26]. In particular our model is completely Hamiltonian and its phase space is not compact.

In order to establish the fluctuation theorem, two ingredients are needed: one needs to prove a large deviation theorem for the ergodic average of the entropy production and establish a symmetry of the large deviation functional. The second part is usually relatively straightforward to establish, at a formal level, since it follows from a symmetry of the generator of the dynamics. This formal derivation for models related to ours can be found in [22] and [19].

The first part, proving the existence of the large deviation functional, involves technical difficulties, in particular if the phase space of the model is not compact. In this case large deviation theorems are established provided the system satisfies very strong ergodic properties (such as hypercontractivity) see e.g. [3, [4, 29]. In addition the entropy production is in general an unbounded observable while standard results of large deviations apply only to bounded observables.

In this paper we show how to treat these difficulties in the model at hand. The techniques we use are based on the construction of Liapunov functions for certain Feynman-Kac semigroups and Perron-Frobenius-like theorem in Banach spaces. We heavily rely on the strong ergodic properties of our model established in [6, 7, 5] and especially in [26].

The Hamiltonian of the model, as in [6], has the form

$$
H=H_{B}+H_{S}+H_{I} .
$$

The two reservoirs of free phonons are described by wave equations in $\mathbf{R}^{d}$ with Hamiltonian

$$
\begin{aligned}
H_{B} & =H\left(\varphi_{L}, \pi_{L}\right)+H\left(\varphi_{R}, \pi_{R}\right), \\
H(\varphi, \pi) & =\frac{1}{2} \int d x\left(|\nabla \varphi(x)|^{2}+|\pi(x)|^{2}\right),
\end{aligned}
$$

where $L$ and $R$ stand for the "left" and "right" reservoirs, respectively. The Hamiltonian describing the chain of length $n$ is given by

$$
\begin{array}{r}
H_{S}(p, q)=\sum_{i=1}^{n} \frac{p_{i}^{2}}{2}+V\left(q_{1}, \cdots, q_{n}\right), \\
V(q)=\sum_{i=1}^{n} U^{(1)}\left(q_{i}\right)+\sum_{i=1}^{n-1} U^{(2)}\left(q_{i}-q_{i+1}\right),
\end{array}
$$

where $\left(p_{i}, q_{i}\right) \in \mathbf{R}^{d} \times \mathbf{R}^{d}$ are the coordinates and momenta of the $i^{t h}$ particle of the chain. The phase space of the chain is $\mathbf{R}^{2 d n}$. The interaction between the chain and the reservoirs occurs at the boundaries only and is of dipole-type

$$
H_{I}=q_{1} \cdot \int d x \nabla \varphi_{L}(x) \rho_{L}(x)+q_{n} \cdot \int d x \nabla \varphi_{R}(x) \rho_{R}(x)
$$


where $\rho_{L}$ and $\rho_{R}$ are coupling functions ("charge densities").

Our assumptions on the anharmonic lattice described by $H_{S}(p, q)$ are the following:

- H1 Growth at infinity: The potentials $U^{(1)}(x)$ and $U^{(2)}(x)$ are $\mathcal{C}^{\infty}$ and grow at infinity like $\|x\|^{k_{1}}$ and $\|x\|^{k_{2}}$ : There exist constants $A_{i}, B_{i}$, and $C_{i}$, $i=1,2$ such that

$$
\begin{aligned}
\lim _{\lambda \rightarrow \infty} \lambda^{-k_{i}} U^{(i)}(\lambda x) & =A_{i}\|x\|^{k_{i}}, \\
\lim _{\lambda \rightarrow \infty} \lambda^{-k_{i}+1} \nabla U^{(i)}(\lambda x) & =A_{i} k_{i}\|x\|^{k_{i}-2} x, \\
\left\|\partial^{2} U^{(i)}(x)\right\| & \leq\left(B_{i}+C_{i} V(x)\right)^{1-\frac{2}{k_{i}}} .
\end{aligned}
$$

Moreover we will assume that

$$
k_{2} \geq k_{1} \geq 2,
$$

so that, for large $\|x\|$ the interaction potential $U^{(2)}$ is "stiffer" than the one-body potential $U^{(1)}$.

- H2 Non-degeneracy: The coupling potential between nearest neighbors $U^{(2)}$ is non-degenerate: For $x \in \mathbf{R}^{d}$ and $m=1,2, \cdots$, let $A^{(m)}(x): \mathbf{R}^{d} \rightarrow$ $\mathbf{R}^{d^{m}}$ denote the linear maps given by

$$
\left(A^{(m)}(x) v\right)_{l_{1} l_{2} \cdots l_{m}}=\sum_{l=1}^{d} \frac{\partial^{m+1} U^{(2)}}{\partial x^{\left(l_{1}\right)} \cdots \partial x^{\left(l_{m}\right)} \partial x^{(l)}}(x) v_{l} .
$$

We assume that for each $x \in \mathbf{R}^{d}$ there exists $m_{0}$ such that

$$
\operatorname{Rank}\left(A^{(1)}(x), \cdots A^{\left(m_{0}\right)}(x)\right)=d .
$$

- H3 Rationality of the coupling: Let $\hat{\rho}_{i}$ denote the Fourier transform of $\rho_{i}$. We assume that

$$
\left|\hat{\rho}_{i}(k)\right|^{2}=\frac{1}{Q_{i}\left(k^{2}\right)},
$$

where $Q_{i}, i \in\{L, R\}$ are polynomials with real coefficients and no roots on the real axis.

We introduce now the temperatures of the reservoirs by choosing initial conditions for the reservoirs. The Hamiltonian of a reservoir is quadratic in $\Psi \equiv(\phi, \pi)$, $H=\langle\Psi, \Psi\rangle / 2$, and therefore the Gibbs measure at temperature $T, d \mu_{T}(\Psi)$ is the Gaussian measure with covariance $T\langle\cdot, \cdot\rangle$. To construct nonequilibrium steady states we assume that 
- The initial conditions $\Psi_{L}=\left(\phi_{L}, \pi_{L}\right)$ and $\Psi_{R}=\left(\phi_{R}, \pi_{R}\right)$ of the reservoirs are distributed according the gaussian Gibbs measures $d \mu_{T_{L}}$ and $d \mu_{T_{R}}$ respectively.

In order to define the heat flow through the bulk of the crystal we consider the energy of the $i^{t h}$ oscillator which we take to be

$$
H_{i}=\frac{p_{i}^{2}}{2}+U^{(1)}\left(q_{i}\right)+\frac{1}{2}\left(U^{(2)}\left(q_{i-1}-q_{i}\right)+U^{(2)}\left(q_{i}-q_{i+1}\right)\right) .
$$

Differentiating $H_{i}$ with respect to time, one finds that

$$
\frac{d H_{i}}{d t}=\Phi_{i-1}-\Phi_{i}
$$

where

$$
\Phi_{i}=\frac{\left(p_{i}+p_{i+1}\right)}{2} \nabla U^{(2)}\left(q_{i}-q_{i+1}\right)
$$

is the heat flow from the $i^{\text {th }}$ to the $(i+1)^{t h}$ particle. We define a corresponding entropy production by

$$
\sigma_{i}=\left(\frac{1}{T_{R}}-\frac{1}{T_{L}}\right) \Phi_{i},
$$

where $T_{R}$ and $T_{L}$ are the temperatures of the reservoirs.

There are other possible definitions of heat flows and corresponding entropy production that one might want to consider. One might, for example, consider the flows $\Phi_{L}, \Phi_{R}$ at the boundary of the chains, and define $\sigma_{b}=-\Phi_{L} / T_{L}-\Phi_{R} / T_{R}$, or one might take other quantities as local energies. But using conservation laws it is easy to see that all these heat flows have the same average in the steady state. Moreover we will show that all the entropy productions have the same large deviations functionals: the exponential part of their fluctuations are identical.

We denote $(p(t), q(t))=\left(p\left(t, p_{0}, q_{0}, \Psi_{L}, \Psi_{R}\right), q\left(t, p_{0}, q_{0}, \Psi_{L}, \Psi_{R}\right)\right)$ as the Hamiltonian flow generated by the Hamiltonian (1D), and consider the ergodic average

$$
\bar{\sigma}_{i}^{t} \equiv \frac{1}{t} \int_{0}^{t} \sigma_{i}(p(s), q(s)) d s .
$$

The quantity $\sigma_{i}(p(s), q(s))$ depends on both the initial conditions of the chain and of the reservoirs which, by assumption, are distributed according to thermal equilibrium. By the ergodic theorem proven in [26] there exists a measure $d \nu$ on $\mathbf{R}^{2 d n}$ such that

$$
\lim _{t \rightarrow \infty} \bar{\sigma}_{i}^{t}=\int \sigma_{i} d \nu .
$$

for all $\left(p_{0}, q_{0}\right)$ and $d \mu_{T_{L}}$ and $d \mu_{T_{R}}$ almost surely. Moreover $\int \sigma_{i} d \nu \equiv\langle\sigma\rangle_{\nu}$ is independent of $i$ and as shown in [7]

$$
\langle\sigma\rangle_{\nu} \geq 0 \text { and }\langle\sigma\rangle_{\nu}=0 \text { if and only if } T_{L}=T_{R} .
$$


Given a set $A \subset \mathbf{R}$, we say that the fluctuations of $\sigma_{i}$ in $A$ satisfy the large deviation principle with large deviation functional $I(w)$ provided

$$
\begin{aligned}
& \inf _{w \in \operatorname{Int}(\mathrm{A})} I(w) \leq \liminf _{t \rightarrow \infty}-\frac{1}{t} \log \mathbf{P}\left\{\bar{\sigma}_{i}{ }^{t} \in A\right\} \leq \\
& \limsup _{t \rightarrow \infty}-\frac{1}{t} \log \mathbf{P}\left\{\bar{\sigma}_{i}{ }^{t} \in A\right\} \leq \inf _{w \in \operatorname{Clos}(\mathrm{A})} I(w) .
\end{aligned}
$$

The study of large deviations for $\sigma_{i}$ is based on the moment generating functionals $e_{i}(\alpha)$ given by

$$
e_{i}(\alpha)=\lim _{t \rightarrow \infty}-\frac{1}{t} \log \int d \mu_{T_{L}} d \mu_{T_{R}} e^{-\alpha \int_{0}^{t} \sigma_{i}(p(s), q(s)) d s} .
$$

The main technical result of this paper is

Theorem 1.1 If

$$
\alpha \in\left(-\frac{T_{\min }}{T_{\max }-T_{\min }}, 1+\frac{T_{\min }}{T_{\max }-T_{\min }}\right),
$$

$e(\alpha) \equiv e_{i}(\alpha)$ is finite and independent of $i$ and the initial conditions $\left(p_{0}, q_{0}\right)$. Moreover e $(\alpha)$ satisfies the relation

$$
e(\alpha)=e(1-\alpha) .
$$

As an application of the Gärtner-Ellis Theorem, see [1], Theorem 2.3.6, we obtain the Gallavotti-Cohen fluctuation theorem.

Theorem 1.2 There is a neighborhood $O$ of the interval $\left[-\langle\sigma\rangle_{\nu},\langle\sigma\rangle_{\nu}\right]$ such that for $A \subset O$ the fluctuations of $\sigma_{i}$ in $A$ satisfy the large deviation principle with a large deviation functional $I(w)$ obeying

$$
I(w)-I(-w)=-w,
$$

i.e., the odd part of $I$ is linear with slope $-1 / 2$.

Theorem 1.2 provides information on the ratio of the probabilities of observing the entropy production to be $w$ and $-w$ : roughly speaking we have

$$
\frac{\mathbf{P}\left\{\bar{\sigma}_{i}^{t} \in(w-\epsilon, w+\epsilon)\right\}}{\mathbf{P}\left\{\bar{\sigma}_{i}{ }^{t} \in(-w-\epsilon,-w+\epsilon)\right\}} \sim e^{w t} .
$$

\section{Fluctuations of the entropy production}

\subsection{Exponential mixing and compactness}

As shown in [6, 26], under condition $\mathbf{H 3}$ the dynamics of the complete system can be reduced to a Markov process on the extended phase space consisting of the 
phase space of the chain $\mathbf{R}^{2 d n}$ and of a finite number of auxiliary variables which we denote as $r$. In the simplest case which we consider here, (corresponding to $\left.\hat{\rho}(k) \sim\left(k^{2}+\gamma^{2}\right)^{-1}\right), r=\left(r_{1}, r_{n}\right) \in \mathbf{R}^{2 d}$ and the resulting equations of motion take the form

$$
\begin{aligned}
\dot{q} & =p \\
\dot{p} & =-\nabla_{q} V-\Lambda^{T} r \\
d r & =(-\gamma r+\Lambda p) d t+(2 \gamma T)^{1 / 2} d \omega .
\end{aligned}
$$

Here $p=\left(p_{1}, \cdots, p_{n}\right)$ and $q=\left(q_{1}, \cdots, q_{n}\right)$ denote the momenta and positions of the particle, $r=\left(r_{1}, r_{n}\right)$ are the auxiliary variables and $\omega$ is a standard $2 d$-dimensional Wiener process. The linear map $\Lambda: \mathbf{R}^{d n} \rightarrow \mathbf{R}^{2 d}$ is given by $\Lambda\left(p_{1}, \ldots, p_{n}\right)=$ $\left(\lambda p_{1}, \lambda p_{n}\right)$ and $T: \mathbf{R}^{2 d} \rightarrow \mathbf{R}^{2 d}$ by $T(x, y)=\left(T_{1} x, T_{n} y\right)$. Here $T_{1} \equiv T_{L}$ and $T_{n} \equiv T_{R}$ are the temperatures of the reservoirs attached to the first and $n^{\text {th }}$ particles respectively, $\gamma$ is the constant appearing in $\hat{\rho}$ and $\lambda$ is a coupling constant equal to $\|\rho\|_{L^{2}}$.

The solution of Eq. (4) $), x(t)=(p(t), q(t), r(t))$ with $x \in X=\mathbf{R}^{2 d(n+1)}$ is a Markov process. We denote $T^{t}$ as the corresponding semigroup

$$
T^{t} f(x)=\mathbf{E}_{x}[f(x(t)],
$$

with generator

$$
L=\gamma\left(\nabla_{r} T \nabla_{r}-r \nabla_{r}\right)+\left(\Lambda p \nabla_{r}-r \Lambda \nabla_{p}\right)+\left(p \nabla_{q}-\left(\nabla_{q} V(q)\right) \nabla_{p}\right),
$$

and we denote $P_{t}(x, d y)$ as the transition probability of the Markov process $x(t)$. In [26] we proved that the Markov process $x(t)$ has smooth transition probabilities, in particular it is strong Feller, and that it is (small-time) irreducible: For any $t>0$, any $x \in X$ and any open set $A \subset X$ we have $P_{t}(x, A)>0$.

There is a natural energy function associated to Eq.(1), given by

$$
G(p, q, r)=\frac{r^{2}}{2}+H(p, q),
$$

which we employ throughout our discussion. In [26] we have constructed a Liapunov function for $x(t)$ from $G$ : Let $t>0$ and $0<\theta<\max \left(T_{1}, T_{n}\right)^{-1}$. Then there exists $E_{0}$ such that for all $E>E_{0}$ there exist functions $\kappa=\kappa(E)<1$ and $b=b(E)<\infty$ such that

$$
T^{t} e^{\theta G}(x) \leq \kappa(E) e^{\theta G}(x)+b(E) \mathbf{1}_{\{G \leq E\}}(x) .
$$

Moreover $\kappa(E)$ can be made arbitrarily small by choosing $E$ sufficiently large, in fact there exist positive constants $c_{1}=c_{1}(\theta, t)$ and $c_{2}=c_{2}(\theta, t)$ such that

$$
\kappa(E) \leq c_{1} e^{-c_{2} E^{2 / k_{2}}} .
$$


By results of 21] it is also shown in 26] that the convergence to the unique stationary state, denoted by $\mu$, occurs exponentially fast: Let $\mathcal{H}_{\infty, \theta}$ denote the Banach space $\left\{f ;\|f\|_{\infty, \theta} \equiv \sup _{x}|f(x)| e^{-\theta G(x)}<\infty\right\}$. Then there exist constants $r>1$ and $R<\infty$

$$
\left|T^{t} f(x)-\int f d \mu\right| \leq R r^{-t}\|f\|_{\infty, \theta} e^{\theta G(x)},
$$

which means that $T^{t}$, acting on $\mathcal{H}_{\infty, \theta}$ has a spectral gap. The methods of [21] are probabilistic and rely on a nice probabilistic construction called splitting as well as coupling arguments and renewal theory.

Under the condition given here, by taking advantage of the fact that the constant $\kappa$ in the Liapunov bound (6) can be made arbitrarily small (this is not assumed in [21]), we can prove stronger ergodic properties and also give a direct analytical proof of Eq. (8).

Besides the Banach space $\mathcal{H}_{\infty, \theta}$ defined above we also consider the Banach space $\mathcal{H}_{\infty, \theta}^{0}=\left\{f,|f| e^{-\theta G} \in C_{0}(X)\right\}$ with norm $\|\cdot\|_{\infty, \theta}\left(C_{0}(X)\right.$ denotes the set of continuous functions which vanish at infinity). Furthermore for $1 \leq p<\infty$ we consider the family of Banach spaces $\mathcal{H}_{p, \theta}=L^{p}\left(X, e^{-p \theta G(x)} d x\right)$ and denote $\|\cdot\|_{p, \theta}$ the corresponding norms.

Theorem 2.1 If $0<\theta T_{i}<1$, the semigroup $T^{t}$ extends to a strongly continuous quasi-bounded semigroup on $\mathcal{H}_{p, \theta}$, for $1 \leq p<\infty$ and on $\mathcal{H}_{\infty, \theta}^{0}$. For any $t>0, T^{t}$ is compact on $\mathcal{H}_{p, \theta}$, for $1<p \leq \infty$ and on $\mathcal{H}_{\infty, \theta}^{0}$.

As an immediate consequence of the spectral properties of positive semigroups [1] and the irreducibility of $x(t)$ we have

Corollary 2.2 The Markov process $x(t)$ has a unique invariant measure $d \mu$ and Eq. (8) holds.

Proof: : Since $T^{t}$ is a Markovian, compact, and irreducible semigroup the eigenvalue 1 is simple with the constant as the eigenfunction. This shows that the Markov process $x(t)$ has a unique invariant measure. Moreover by the cyclicity properties of the spectrum of a positive semigroup [11], and by the compactness of $T^{t}$, there are no other eigenvalues of modulus 1. Eq. (8) follows immediately.

Proof of Theorem 2.1 In 26], Lemma 3.6, we showed that for some constant $C$ $T^{t} e^{\theta G} \leq e^{c t} e^{\theta G}$ provided $\theta T_{i}<1$ (see also Lemma 2.9 below). Therefore for $f C^{\infty}$ with compact support we have, using Ito's and Girsanov's formulas

$$
\begin{aligned}
e^{-\theta G} T^{t} e^{\theta G} f(x) & =\mathbf{E}_{x}\left[e^{\theta(G(x(t))-G(x))} f(x)\right] \\
& =\mathbf{E}_{x}\left[e^{\theta \int_{0}^{t} \gamma\left(\operatorname{Tr}(T)-r^{2}\right) d s+\theta \int_{0}^{t} \sqrt{2 \gamma T} r d \omega(s)} f(x(t))\right] \\
& =\mathbf{E}_{x}\left[e^{\gamma \theta \operatorname{Tr}(T)+\gamma \tilde{r}\left(\theta^{2} T-\theta\right) \tilde{r}} f(\tilde{x}(t))\right]
\end{aligned}
$$


where $\tilde{x}$ is the process with generator

$$
\tilde{L}_{\theta}=L+2 \gamma \theta r T \nabla_{r}
$$

A computation shows that $\tilde{L}_{\theta}^{T} 1=\gamma \operatorname{Tr}(1-2 \theta T)$. Standard arguments show then that the semigroup associated with the process $\tilde{x}$ extends to a quasi-bounded and strongly continuous semigroup on $L^{p}(d x), 1 \leq p<\infty$ and on $C_{0}(X)$. Using the assumption that $\theta T_{i}<1$ and Feynman-Kac formula we see that $e^{-\theta G} T^{t} e^{\theta G}$ extends too to a quasi-bounded and strongly continuous semigroup on $L^{p}(d x)$, $1 \leq p<\infty$ and on $C_{0}(X)$. This implies immediately that $T^{t}$ extends to a strongly continuous semigroup on $\mathcal{H}_{p, \theta}, 1 \leq p<\infty$ and $\mathcal{H}_{\infty, \theta}^{0}$. The computation above also shows that $T^{t}$ extends to a quasi-bounded semigroup on $\mathcal{H}_{\infty, \theta}$.

We first prove the compactness of $T^{t}$ for $\mathcal{H}_{\infty, \theta}$. If $f \in \mathcal{H}_{\infty, \theta}$ then $|f(x)| \leq$ $\|f\|_{\infty, \theta} e^{\theta G(x)}$ and by (6) and (7) we obtain

$$
\begin{aligned}
\left|\mathbf{1}_{G \geq E} T^{t} f(x)\right| & \leq e^{\theta G(x)} \sup _{\{y: G(y) \geq E\}} \frac{\left|T^{t} f(y)\right|}{e^{\theta G(y)}} \\
& \leq e^{\theta G(x)}\|f\|_{\infty, \theta} \sup _{\{y: G(y) \geq E\}} \frac{T^{t} e^{(\theta G(y))}}{e^{\theta G(y)}} \\
& \leq \kappa(E) e^{\theta G(x)}\|f\|_{\infty, \theta} .
\end{aligned}
$$

From the bounds (9) and (7) we conclude that the operator $\mathbf{1}_{\{G \geq E\}} T^{t}$ converges uniformly to 0 in $\mathcal{H}_{\infty, \theta}$ as $E \rightarrow \infty$. The semigroup $T^{t}$ has a $C^{\infty}$ kernel since it is generated by a hypoelliptic operator see [26], Proposition 4.1, so, by the ArzelaAscoli theorem $\mathbf{1}_{\{G \leq E\}} T^{t / 2} \mathbf{1}_{\{G \leq E\}}$ is compact, for any $E$. Therefore we obtain

$$
T^{t}=\lim _{E \rightarrow \infty} \mathbf{1}_{\{G \leq E\}} T^{t / 2} \mathbf{1}_{\{G \leq E\}} T^{t / 2}
$$

where the limit is in the norm sense from (9) above, i.e., $T^{t}$ is the uniform limit of compact operators, hence is compact.

The compactness of $T^{t}$ for $\mathcal{H}_{\infty, \theta}^{0}$ follows from the same argument. In fact by Eq. (7), for any $t>0, T^{t} \mathcal{H}_{\infty, \theta} \subset \mathcal{H}_{\infty, \theta}^{0}$.

To prove the compactness of $T^{t}$ on $\mathcal{H}_{p \theta}, 1<p<\infty$, we note that

$$
\begin{aligned}
\left|T^{t} f(x)\right| & =\left|\mathbf{E}_{x}[f(x(t))]\right| \\
& =\left|\mathbf{E}_{x}\left[e^{\frac{\theta}{q} G(x(t))} e^{-\frac{\theta}{q} G(x(t))} f(x(t))\right]\right| \\
& \leq\left(\mathbf{E}_{x}\left[e^{\theta G(x(t))}\right]\right)^{1 / q}\left(\mathbf{E}_{x}\left[e^{-\frac{p \theta}{q} G(x(t))} f^{p}(x(t))\right]\right)^{1 / p}
\end{aligned}
$$

Thus using the bound $(\nabla)$ and the fact that $T^{t}$ is quasi-bounded on $\mathcal{H}_{1, \theta}$ we obtain

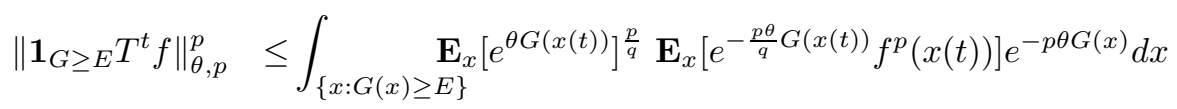




$$
\begin{aligned}
& \leq \sup _{\{x: G(x) \geq E\}}\left(\frac{\mathbf{E}_{x}\left[e^{\theta G(x(t))}\right]}{e^{\theta G(x)}}\right)^{\frac{p}{q}}\left\|T^{t}\left(e^{-\frac{p \theta}{q} G} f^{p}\right)\right\|_{1, \theta} \\
& \leq \kappa(E)^{\frac{p}{q}} e^{c t}\left\|e^{-\frac{p \theta}{p} G} f^{p}\right\|_{1, \theta} \\
& =\kappa(E)^{\frac{p}{q}} e^{c t}\|f\|_{\theta, p}^{p} .
\end{aligned}
$$

As in the case $p=\infty$, we conclude from the bound (7) that the operator $\mathbf{1}_{G \geq E} T^{t}$ converges uniformly to 0 in $\mathcal{H}_{p, \theta}$ as $E \rightarrow \infty$. Using that the kernel of $\mathbf{1}_{\{G \leq E\}} T^{t} \mathbf{1}_{\{G \leq E\}}$ is bounded, we conclude that $T^{t}$ is compact on $\mathcal{H}_{p, \theta}$ for $1<p<$ $\infty$.

\subsection{Heat flow and generating functionals}

In order to define the heat flows we note that we have

$$
\frac{d}{d t} T^{t} H=L T^{t} H=T^{t}(-r \Lambda p)=T^{t}\left(-\lambda r_{1} p_{1}-\lambda r_{n} p_{n}\right) .
$$

Hence we identify $\Phi_{0} \equiv-\lambda r_{1} p_{1}$ as the observable describing the heat flow from the left reservoir into the chain and $\Phi_{n} \equiv \lambda r_{n} p_{n}$ as the heat flow from the chain into the right reservoir. As in the introduction we define the energy $H_{i}$ of the $i^{\text {th }}$ oscillators by Eq.(2), for $i \leq 2 \leq n-1$, and

$$
\begin{aligned}
& H_{1}=\frac{p_{1}^{2}}{2}+U^{(1)}\left(q_{1}\right)+\frac{1}{2} U^{(2)}\left(q_{1}-q_{2}\right), \\
& H_{n}=\frac{p_{n}^{2}}{2}+U^{(1)}\left(q_{n}\right)+\frac{1}{2} U^{(2)}\left(q_{n-1}-q_{n}\right) .
\end{aligned}
$$

With the heat flows $\Phi_{i}, i=1, \cdots, n$, defined as in Eq. (3) we have

$$
L H_{i}=\Phi_{i-1}-\Phi_{i}, \quad i=1, \cdots, n .
$$

and we define the entropy productions $\sigma_{i}, i=0, \cdots, n$ by

$$
\sigma_{i}=\left(\frac{1}{T_{1}}-\frac{1}{T_{n}}\right) \Phi_{i} \quad i=0, \cdots, n .
$$

We now provide several identities involving the generator of the dynamics and the entropy production, which will play a crucial role in our subsequent analysis.

Lemma 2.3 Let the function $R_{i}, i=0, \cdots, n$ be given by

$$
R_{i}=\frac{1}{T_{1}}\left(\frac{r_{1}^{2}}{2}+\sum_{k=1}^{i} H_{i}(p, q)\right)+\frac{1}{T_{n}}\left(\sum_{k=i+1}^{n} H_{i}(p, q)+\frac{r_{n}^{2}}{2}\right) .
$$

Then we have

$$
\sigma_{i}=r T^{-1} r-\operatorname{Tr}(I)+L R_{i}
$$


Proof: This is a straightforward computation.

Remark 2.4 This shows that, up to a derivative, all the entropy productions are equal to the quantity $r T^{-1} r-\operatorname{Tr} \gamma I$ which is independent of $i$ and involves only the $r$-variables.

Let $L^{T}$ be the formal adjoint of the operator $L$ given by Eq. (5)

$$
L^{T}=\gamma\left(\nabla_{r} T \nabla_{r}+r \nabla_{r}\right)-\left(\Lambda p \nabla_{r}-r \Lambda \nabla_{p}\right)-\left(p \nabla_{q}-\left(\nabla_{q} V(q)\right) \nabla_{p}\right),
$$

and let $J$ be the time reversal operator which changes the sign of the momenta of all particles, $J f(p, q, r)=f(-p, q, r)$.

The following identities can be regarded as operator identities on $\mathcal{C}^{\infty}$ functions. That the left and right side of Eq. (14) actually generate semigroups for some non trivial domain of $\alpha$ is a non trivial result which we will discuss in Section 2.3.

Lemma 2.5 We have the operator identities

$$
e^{R_{i}} J L^{T} J e^{-R_{i}}=L-\sigma_{i},
$$

and also for any constant $\alpha$

$$
e^{-R_{i}} J\left(L^{T}-\alpha \sigma_{i}\right) J e^{R_{i}}=L-(1-\alpha) \sigma_{i} .
$$

Proof: We write the generator $L$ as $L=L_{0}+L_{1}$ with

$$
\begin{aligned}
& L_{0}=\gamma\left(\nabla_{r} T \nabla_{r}-r \nabla_{r}\right) \\
& L_{1}=\left(\Lambda p \nabla_{r}-r \Lambda \nabla_{p}\right)+\left(p \nabla_{q}-\left(\nabla_{q} V(q)\right) \nabla_{p}\right) .
\end{aligned}
$$

Since $L_{1}$ is a first order differential operator we have

$$
e^{-R_{i}} L_{1} e^{R_{i}}=L_{1}+L_{1} R_{i}=L_{1}+\sigma_{i} .
$$

Using that $\nabla_{r} R_{i}=T^{-1} r$ we obtain

$$
\begin{aligned}
e^{-R_{i}} L_{0} e^{R_{i}} & =e^{-R_{i}} \gamma\left(\nabla_{r}-T^{-1} r\right) T \nabla_{r} e^{R_{i}} \\
& =\gamma \nabla_{r} T\left(\nabla_{r}+T^{-1} r\right)=L_{0}^{T} .
\end{aligned}
$$

This gives

$$
e^{-R_{i}} L e^{R_{i}}=L_{0}^{T}+L_{1}+\sigma_{i}=J L^{T} J+\sigma_{i},
$$

which is Eq. (13). Since $J \sigma_{i} J=-\sigma_{i}$, Eq. (14) follows immediately from Eq. (13).

Remark 2.6 In the equilibrium situation, i.e., for $T_{1}=T_{n}=T$, Eq. (14) is

$$
e^{G / T} J L^{T} J e^{-G / T}=L,
$$


which is simply detailed balance. Eq. (14) can be interpreted in path space in the following manner [18: Let $\Pi$ denote the time-reversal in path space on the time interval $[0, t]: \Pi(p(s), q(s), r(s))=(-p(t-s), q(t-s), r(t-s))$ and let $d P$ denote the measure on $\mathcal{C}([0, t], X)$ induced by $x(t)$. Then Eq. (14) implies that

$$
\frac{d P \circ \Pi}{d P}=e^{R_{i}(x(t))-R_{i}(x(0))-\int_{0}^{t} \sigma_{i}(x(s)) d s} .
$$

This formula exhibits the fact that the lack of microscopic reversibility is intimately related to the entropy production.

We now turn to the study of the large deviations. As shown in [26] the Markov process $x(t)$ is ergodic. In order to study the large deviations of $t^{-1} \int_{0}^{t} \sigma_{i}(x(s)) d s$ we consider the moment generating functionals

$$
\Gamma_{x}^{i}(t, \alpha)=\mathbf{E}_{x}\left[e^{-\alpha \int_{0}^{t} \sigma_{i}(x(s)) d s}\right] .
$$

Formally the Feynman-Kac formula gives $\Gamma_{x}^{i}(t, \alpha)=e^{t\left(L-\alpha \sigma_{i}\right)} 1(x)$, but since $\sigma_{i}$ is not bounded, nor even relatively bounded by $L$, it is not obvious that $\Gamma_{x}^{i}(t, \alpha)$ exists for $\alpha \neq 0$. Our goal is to prove that $\Gamma_{x}^{i}(t, \alpha)$ exists and that the limit

$$
e(\alpha) \equiv \lim _{t \rightarrow \infty}-\frac{1}{t} \log \Gamma_{x}^{i}(t, \alpha)
$$

exists and is finite in a neighborhood of the interval $[0,1]$, and is independent of $i$ and of the initial condition $x$.

The technical difficulty in proving the existence of the limit (17) lies in the fact that the functions $\sigma_{i}$ are unbounded. Standard large deviation theorems for Markov processes (see e.g. [3, [, 29]) are proven usually under strong ergodic properties for bounded functions and are not directly applicable. Large deviations for unbounded functions are considered in [1] for discrete time countable state space Markov chains under conditions which amount in our case to $\sigma=o(G)$. In our case this is clearly not satisfied since, in general $\sigma$ is not bounded by $G$.

But the $\sigma_{i}$ are very special observables, in particular they are intimately linked with the dynamics as shown by the identities Eqs.(13) and (14). The next lemma displays another identity which will be important in our analysis.

Lemma 2.7 We have the identity

$$
L-\alpha \sigma_{i}=e^{\alpha R_{i}} \bar{L}_{\alpha} e^{-\alpha R_{i}},
$$

where

$$
\bar{L}_{\alpha}=\tilde{L}_{\alpha}-\left(\left(\alpha-\alpha^{2}\right) \gamma r T^{-1} r-\alpha \operatorname{Tr}(\gamma I)\right)
$$

and

$$
\tilde{L}_{\alpha}=L+2 \alpha \gamma r \nabla_{r}
$$


Proof: As in Lemma 2.5 we write the generator $L$ as $L=L_{0}+L_{1}$, see Eqs.(16) and (15). Since $L_{1}$ is a first order differential operator we have

$$
e^{-\alpha R_{i}} L_{1} e^{\alpha R_{i}}=L_{1}+\alpha\left(L_{1} R_{i}\right)=L_{1}+\alpha \sigma_{i} .
$$

Using that $\nabla_{r} R_{i}=T^{-1} r$ is independent of $i$ we find that

$$
\begin{aligned}
e^{-\alpha R_{i}} L_{0} e^{\alpha R_{i}} & =\gamma\left(\left(\nabla_{r}+\alpha T^{-1} r\right) T\left(\nabla_{r}+\alpha T^{-1} r\right)-r\left(\nabla_{r}+\alpha T^{-1} r\right)\right) \\
& =L_{0}+\alpha \gamma\left(r \nabla_{r}+\nabla_{r} r\right)+\left(\alpha^{2}-\alpha\right) \gamma r T^{-1} r \\
& =L_{0}+2 \alpha \gamma r \nabla_{r}+\left(\alpha^{2}-\alpha\right) \gamma r T^{-1} r+\alpha \operatorname{Tr} \gamma I
\end{aligned}
$$

Combining Eqs. (21) and (22) gives the desired result.

Remark 2.8 The identity (18) shows that all operators $L-\alpha \sigma_{i}$ are conjugate to the same operator $\bar{L}_{\alpha}$. This will be the key element to prove that $e(\alpha)$ is independent of $i$. Furthermore it can be seen from Eqs. (19) and (20) that $\bar{L}_{\alpha}$ has the form of $L$ plus a perturbation which is a quadratic form in $r$ and $\nabla_{r}$. Such a perturbation is indeed nicer than $\alpha \sigma_{i}$. Also it should be noted that $\tilde{L}_{\alpha}$ has very much the same form as the operator $L$ : they differ only by the coefficient in front of the term $r \nabla_{r}$. This fact will allow us to use several results on $L$ obtained in [26.

\subsection{Liapunov Function for Feynman-Kac Semigroups}

At this point we begin the study of $\bar{L}_{\alpha}$ as the generator of a semigroup.

Proposition 2.9 If $\theta$ and $\alpha$ satisfy the condition

$$
-\alpha<\theta T_{i}<1-\alpha,
$$

then there exists a constant $C=C(\alpha, \theta)$ such that $e^{t \bar{L}_{\alpha}} e^{\theta G}(x) \leq e^{C t} e^{\theta G}(x)$.

Proof: We note first that $\tilde{L}_{\alpha}$, defined in Eq. (19), for all $\alpha \in \mathbf{R}$, is the generator of a Markov process which we denote as $\tilde{x}(t)$. Indeed we have that

$$
\tilde{L}_{\alpha} G(x)=\operatorname{Tr}(\gamma T)-(1+2 \alpha) r^{2} \leq C_{1}+C_{2} G(x)
$$

Since $G$ grows at infinity, $G$ is a Liapunov function for $\tilde{x}(t)$ and a standard argument [16 shows that the Markov process $\tilde{x}(t)$ is non-explosive. Furthermore we have the bound

$$
\begin{aligned}
& \bar{L}_{\alpha} \exp \theta G(x)= \\
& =\exp \theta G(x) \gamma\left[\operatorname{Tr}(\theta T+\alpha I)+r\left(\theta^{2} T-(1-2 \alpha) \theta-\alpha(1-\alpha) T^{-1}\right) r\right] \\
& \leq C \exp \theta G(x)
\end{aligned}
$$


provided $\alpha$ and $T_{i}, i=1, n$ satisfy the inequality

$$
\theta^{2} T_{i}-(1-2 \alpha) \theta-\alpha(1-\alpha) T_{i}^{-1} \leq 0,
$$

or

$$
-\alpha<\theta T_{i}<1-\alpha .
$$

We denote $\sigma_{R}$ as the exit time from the set $\{G(x)<R\}$, i.e., $\sigma_{R}=\inf \{t \geq$ $0, G(\tilde{x}(t)) \geq R\}$. If the initial condition $x$ satisfies $G(x)=E<R$, we denote by $\tilde{x}_{R}(t)$ the process which is stopped when it exits $\{G(x)<R\}$, i.e., $\tilde{x}_{R}(t)=\tilde{x}(t)$ for $t<\sigma_{R}$ and $\tilde{x}_{R}(t)=x\left(\sigma_{R}\right)$ for $t \geq \sigma_{R}$. Finally we set $\sigma_{R}(t)=\min \left\{\sigma_{R}, t\right\}$.

By Eq. (24), the function $W(t, x)=e^{-C t} e^{\theta G(x)}$ satisfies the inequality $\left(\partial_{t}+\right.$ $\left.\bar{L}_{\alpha}\right) W(t, x) \leq 0$ and applying Ito's formula with stopping time to the function $W(t, x)$ we obtain

$$
\mathbf{E}_{x}\left[e^{-\int_{0}^{\sigma_{R}(t)}\left(\left(\alpha-\alpha^{2}\right) \gamma \tilde{r} T^{-1} \tilde{r}-\alpha \operatorname{Tr}(\gamma I)\right) d s} e^{\theta G\left(\tilde{x}\left(\sigma_{R}(t)\right)\right)} e^{-C \sigma_{R}(t)}\right]-e^{\theta G(x)} \leq 0,
$$

and thus

$$
\mathbf{E}_{x}\left[e^{-\int_{0}^{\sigma_{R}(t)}\left(\left(\alpha-\alpha^{2}\right) \gamma \tilde{r} T^{-1} \tilde{r}-\alpha \operatorname{Tr}(\gamma I)\right) d s} e^{\theta G\left(\tilde{x}\left(\sigma_{R}(t)\right)\right)}\right] \leq e^{C t} e^{\theta G(x)} .
$$

Since the Markov process $\tilde{x}(t)$ is non-explosive $G\left(\tilde{x}_{R}(t)\right) \rightarrow G(\tilde{x}(t))$ almost surely as $R \rightarrow \infty$, so by the Fatou lemma we have

$$
e^{t \bar{L}_{\alpha}} e^{\theta G}(x) \leq e^{C t} e^{\theta G}(x) .
$$

This concludes the proof of Lemma 2.9.

The next three theorems are all consequences of the fact that $\tilde{L}_{\alpha}$ is the generator of a Markov process which is similar to the process generated by $L$ : Indeed $L$ and $\tilde{L}_{\alpha}$ differ only by the coefficient in front of the $r \nabla_{r}$ term. Therefore repeating the proofs of [26] we obtain

Theorem 2.10 The semigroup $e^{t \bar{L}_{\alpha}}$ has a smooth kernel $q_{\alpha}(t, x, y)$ which belongs to $\mathcal{C}^{\infty}((0, \infty) \times X \times X)$.

Proof: The operator $\tilde{L}_{\alpha}$ satisfies the same Hörmander-type condition that $L$ proven in [26], Proposition 4.1.The result follows then from [12] or [23].

Theorem 2.11 The semigroup $e^{t \bar{L}_{\alpha}}$ is positivity improving for all $t>0$.

Proof: The semigroup $e^{t \tilde{L}_{\alpha}}$ is shown to be irreducible exactly as $e^{t L}$, see [7] 26] using explicit computation and the Support Theorem of [28]. The statement follows then from the Feynman-Kac formula.

As is apparent from the form of $\bar{L}_{\alpha}$ we will need estimates on the observable $r^{2}$ in the sequel. Such estimates were also crucial in [26] for the construction of a Liapunov function. 
Theorem 2.12 Let $0 \leq \alpha<1 / k_{2}$ and let $t_{E}=E^{1 / k_{2}-1 / 2}$. There exists a set of paths

$$
S\left(x, E, t_{E}\right) \subset\left\{f \in \mathcal{C}\left(\left[0, t_{E}\right], X\right) ; f(0)=x, G(x)=E\right\},
$$

and constants $E_{0}<\infty$ and $A, B, C>0$ such that for $E>E_{0}$

$$
\mathbf{P}\left\{\tilde{x} \in S\left(x, E, t_{E}\right)\right\} \geq 1-A e^{-B E^{2 \alpha+1 / 2-1 / k_{2}}},
$$

and

$$
\int_{0}^{t_{E}} \tilde{r}^{2}(s) d s \geq C E^{3 / k_{2}-1 / 2}, \quad \text { if } \quad \tilde{x} \in S\left(x, E, t_{E}\right) .
$$

Proof: The proof is exactly as in [26]. One first sets $T_{1}=T_{n}=0$ in the equations of motion and then, by a scaling argument, Theorem 3.3 of [26], one shows that the deterministic trajectory satisfies the estimate (25). Then one shows, see Proposition 3.7 and Corollary 3.8 of [26], that the overwhelming majority of the random trajectories follows very closely the deterministic ones. We refer the reader to 26] for further details.

Remark 2.13 For large energy $E$, paths satisfying the bound (25) have a very high probability. From Eq. (25) we obtain that, on a time interval of order 1,

$$
\int_{0}^{t} \tilde{r}^{2}(s) \geq C E^{2 / k_{2}}
$$

for an overwhelming majority of the paths.

Theorem 2.14 Let $t>0$ be fixed and suppose that $\alpha$ and $\theta$ satisfy the condition $E q$.20. There exist a constant $E_{0}$ and functions $\kappa(E)$ and $b(E)$ such that for $E>E_{0}$

$$
e^{t \bar{L}_{\alpha}} e^{\theta G}(x) \leq \kappa(E) e^{\theta G(x)}+b(E) \mathbf{1}_{\{G \leq E\}}(x) .
$$

Moreover there exist constants $c_{1}$ and $c_{2}$ such that

$$
\kappa(E) \leq c_{1} e^{-c_{2} E^{2 / k_{2}}} .
$$

Proof: By Proposition 2.9 the function $e^{t \bar{L}_{\alpha}} e^{\theta G}(x)$ is bounded on any compact set. Therefore to show (26) it suffices to show that

$$
\sup _{\{x: G(x)>E\}} \mathbf{E}_{x}\left[e^{-\int_{0}^{t}\left(\alpha(1-\alpha) \gamma \tilde{r} T^{-1} \tilde{r}-\alpha \operatorname{Tr}(\gamma I)\right) d s} e^{\theta(G(\tilde{x}(t))-G(\tilde{x}))}\right] \leq \kappa(E) .
$$

Using Ito's formula we have

$$
G(\tilde{x}(t))-G(x)=\int_{0}^{t} \gamma\left(\operatorname{Tr}(T)-\tilde{r}^{2}\right) d s+\int_{0}^{t} \sqrt{2 \gamma T} \tilde{r} d \omega(s),
$$


and thus we obtain

$$
\begin{array}{r}
\mathbf{E}_{x}\left[e^{-\int_{0}^{t}\left(\alpha(1-\alpha) \gamma \tilde{r} T^{-1} \tilde{r}-\alpha \operatorname{Tr}(\gamma I)\right) d s} e^{\theta(G(x(t))-G(x))}\right] \\
=e^{t \gamma \operatorname{Tr}(\theta T+\alpha I)} \mathbf{E}_{x}\left[e^{-\int_{0}^{t} \tilde{r}\left(\alpha(1-\alpha) \gamma T^{-1}-\gamma \theta(1-2 \alpha)\right) \tilde{r} d s} \times\right. \\
\left.\times e^{\int_{0}^{t} \theta \sqrt{2 \gamma T} \tilde{r} d \omega}\right] .
\end{array}
$$

Using the Hölder's inequality we find that the expectation on the r.h.s of Eq. (27) can be estimated by

$$
\begin{aligned}
& \mathbf{E}_{x}\left[e^{-q \int_{0}^{t} \tilde{r}\left(\alpha(1-\alpha) \gamma T^{-1}-\gamma \theta(1-2 \alpha)\right) \tilde{r} d s} e^{\frac{q p \theta^{2}}{2} \int_{0}^{t}(\sqrt{2 \gamma T} \tilde{r})^{2} d s}\right]^{1 / q} \\
& \quad \times \mathbf{E}_{x}\left[e^{-\frac{p^{2} \theta^{2}}{2} \int_{0}^{t}(\sqrt{2 \gamma T} \tilde{r})^{2} d s} e^{\left.p \int_{0}^{t} \theta(2 \gamma T)^{1 / 2} \tilde{r} d \omega\right)}\right]^{1 / p} \\
& =\mathbf{E}_{x}\left[e^{-q \gamma \int_{0}^{t} \tilde{r}\left(\alpha(1-\alpha) T^{-1}-\theta(1-2 \alpha)+p \theta^{2} T\right) \tilde{r} d s}\right]^{1 / q} .
\end{aligned}
$$

where we have used that the second factor is the expectation of a martingale with expectation 1.

If $\theta$ and $\alpha$ satisfy the condition (23), then, by choosing $p$ sufficiently close to 1, the quadratic form in the right side of Eq. (28) is negative definite. Using Theorem 2.12 as in Theorem 3.11 of [26] we obtain

$$
\begin{aligned}
& \sup _{x \in U^{C}} \mathbf{E}_{x}\left[e^{-\int_{0}^{t}\left(\alpha(1-\alpha) \tilde{r} T^{-1} \tilde{r}-\alpha \operatorname{Tr}(\gamma I)\right) d s} e^{\theta(G(x(t))-G(x))}\right] \\
& \leq e^{\gamma \operatorname{Tr}(\theta T+\alpha I)} e^{-C E^{2 / k_{2}} \gamma \operatorname{Tr}\left(\alpha(1-\alpha) T^{-1}-(1-2 \alpha) \theta+p \theta^{2} T\right)} \\
& \leq c_{1} e^{-c_{2} E^{2 / k_{2}}} .
\end{aligned}
$$

and this concludes the proof of Theorem 2.14.

As in Theorem 2.1 we obtain

Theorem 2.15 If $\alpha$ and $\theta$ satisfy the condition Eq. (2O), then $e^{t \bar{L}_{\alpha}}$ extends to a strongly continuous quasi-bounded semigroup on $\mathcal{H}_{p, \theta}$ for $1 \leq p<\infty$ and on $\mathcal{H}_{\infty, \theta}^{0}$. Moreover $e^{t \bar{L}_{\alpha}}$ is compact on $\mathcal{H}_{p, \theta}, 1<p \leq \infty$ and on $\mathcal{H}_{\infty, \theta}^{0}$.

Proof: The proof is a repetition of the proof of Theorem 2.1 and is left to the reader.

As a consequence of Theorem 2.15 and of the theory of semigroup of positive operators [1] we obtain 
Theorem 2.16 If

$$
\alpha \in\left(-\frac{T_{\min }}{T_{\max }-T_{\min }}, 1+\frac{T_{\min }}{T_{\max }-T_{\min }}\right),
$$

then

$$
e(\alpha)=\lim _{t \rightarrow \infty}-\frac{1}{t} \log \Gamma_{x}^{i}(t, \alpha)
$$

exists, is finite and independent both of $i$ and $x$.

Proof: By Theorem 2.15, $e^{t \bar{L}_{\alpha}}$ generates a strongly continuous semigroup on $\mathcal{H}_{\infty, \theta}^{0}$ if

$$
-\alpha<\theta T_{i}<1-\alpha .
$$

If $\alpha \leq 0$, this implies that $|\alpha|<\theta T_{\min }<\theta T_{\max }<1+|\alpha|$ and so the set of $\theta$ we can choose is non-empty provided

$$
\alpha>-\frac{T_{\min }}{T_{\max }-T_{\min }} .
$$

If $0<\alpha<1$, we can always find $\theta$ such that (29) is satisfied. Finally if $\alpha>1$ then (29) implies that that

$$
\alpha<1+\frac{T_{\min }}{T_{\max }-T_{\min }} .
$$

By the definition of $R_{i}$, Eq. (10), $e^{-\alpha R_{i}} \in \mathcal{H}_{\infty, \theta}^{0}$ since $-\alpha+\theta T_{i}<0$. Using now Lemma 2.7, we see that $\Gamma_{x}^{i}(t, \alpha)$ exists and is given by

$$
\Gamma_{x}^{i}(t, \alpha)=e^{t(L-\alpha \sigma)} 1(x)=e^{\alpha R_{i}} e^{t \bar{L}_{\alpha}} e^{-\alpha R_{i}}(x) .
$$

From Theorem 2.11 the semigroup $e^{t \bar{L}_{\alpha}}$ is an irreducible semigroup of compact operators on the Banach space $\mathcal{H}_{\infty, \theta}^{0}$. From the cyclicity properties of the spectrum of irreducible operators and from the compactness it follows (see [11], Chapter C-III) that there is exactly one eigenvalue $e^{-t e(\alpha)}$ with maximal modulus and this eigenvalue is real and simple. The corresponding eigenfunction $f_{\alpha}$ is strictly positive and we denote as $P_{\alpha}$ the one-dimensional projection on the eigenspace spanned by $f_{\alpha}$. In particular if $g \geq 0$, then $P_{\alpha} g(x)>0$.

From compactness it follows that the complementary projection $\left(1-P_{\alpha}\right)$ satisfies the bound

$$
\left|e^{t \bar{L}_{\alpha}}\left(1-P_{\alpha}\right) f(x)\right| \leq C e^{-t d(\alpha)}\|f\|_{\infty, \theta} e^{\theta G(x)} .
$$

for some constants $C>0$ and $d(\alpha)>e(\alpha)$ and for all $t>0$. 
From Lemma 2.7 and Eq. (30) we obtain, for all $x \in X$, that

$$
\begin{aligned}
\lim _{t \rightarrow \infty}-\frac{1}{t} \log \Gamma_{x}^{i}(t, \alpha) \\
=\lim _{t \rightarrow \infty}-\frac{1}{t} \log e^{t\left(L-\alpha \sigma_{i}\right)} 1(x)=\lim _{t \rightarrow \infty}-\frac{1}{t} \log e^{\alpha R_{i}} e^{t \bar{L}_{\alpha}} e^{-\alpha R_{i}}(x) \\
=\lim _{t \rightarrow \infty}\left(-\frac{1}{t} \alpha R_{i}(x)\right)+e(\alpha) \\
\quad+\lim _{t \rightarrow \infty}-\frac{1}{t} \log \left(P_{\alpha} e^{-\alpha R_{i}}(x)+e^{t e(\alpha)} e^{t \bar{L}_{\alpha}}\left(1-P_{\alpha}\right) e^{-\alpha R_{i}}(x)\right) \\
=e(\alpha) .
\end{aligned}
$$

This concludes the proof of Theorem 2.16.

Using now the identity (14) we can prove the symmetry of $e(\alpha)$. Theorem 1.1 is then an immediate consequence of the following result.

\section{Theorem 2.17 If}

$$
\alpha \in\left(-\frac{T_{\min }}{T_{\max }-T_{\min }}, 1+\frac{T_{\min }}{T_{\max }-T_{\min }}\right),
$$

then

$$
e(\alpha)=e(1-\alpha) .
$$

Proof: If $\alpha$ is in the interval (32) and $-\alpha<\theta T_{i}<1-\alpha$ then $e^{t \bar{L}_{\alpha}}$ is a strongly continuous compact semigroup on $\mathcal{H}_{\infty, \theta}^{0}$. By Lemma 2.7

$$
e^{t\left(L-\alpha \sigma_{i}\right)}=e^{\alpha R_{i}} e^{t \bar{L}_{\alpha}} e^{-\alpha R_{i}}
$$

is also a strongly continuous compact semigroup on the Banach space $\mathcal{H}_{\infty, \theta, \alpha}^{0}=$ $\left\{f ;|f| e^{-\theta G+\alpha R_{i}} \in C_{0}(x)\right\}$ with the norm $\|f\|_{\infty, \theta, \alpha}=\sup |f| e^{\theta G+\alpha R_{i}}$.

The dual semigroup $\left(e^{t\left(L-\alpha \sigma_{i}\right)}\right)^{*}$ is a compact semigroup on the Banach space (of measures) $\left(\mathcal{H}_{\infty, \theta, \alpha}^{0}\right)^{*}$. By Theorem $2.11\left(e^{t\left(L-\alpha \sigma_{i}\right)}\right)^{*}$ maps $\left(\mathcal{H}_{\infty, \theta, \alpha}^{0}\right)^{*}$ into measures with smooth densities and on densities $\left(e^{t\left(L-\alpha \sigma_{i}\right)}\right)^{*}$ acts as

$$
\left(e^{t\left(L-\alpha \sigma_{i}\right)}\right)^{*}(\rho(x) d x)=\left(e^{t\left(L^{T}-\alpha \sigma_{i}\right)} \rho(x)\right) d x .
$$

By Lemma 2.5 we have

$$
e^{-R_{i}} e^{t\left(L-(1-\alpha) \sigma_{i}\right)} 1(x)=J e^{t\left(L^{T}-\alpha \sigma_{i}\right)} J e^{-R}(x) .
$$

Since $-\alpha<\theta T_{i}<1-\alpha, e^{-R_{i}}$ is a density of a measure in $\left(\mathcal{H}_{\infty, \theta, \alpha}^{0}\right)^{*}$. Since $\left(e^{t\left(L-\alpha \sigma_{i}\right)}\right)^{*}$ is compact and irreducible with spectral radius $e(\alpha)$ we obtain using 
Eq. (33)

$$
\begin{aligned}
e(\alpha) & =\lim _{t \rightarrow \infty}-\frac{1}{t} \log \left\|J\left(e^{t\left(L^{T}-\alpha \sigma_{i}\right)} J e^{-R_{i}}\right) d x\right\| \\
& =\lim _{t \rightarrow \infty}-\frac{1}{t} \log \left(\sup _{f \leq e^{\theta G+\alpha R_{i}}} \int f e^{-R_{i}} e^{t\left(L-(1-\alpha) \sigma_{i}\right)} 1 d x\right), \\
& =e(1-\alpha) .
\end{aligned}
$$

In the last equality we have used Theorem 2.16 and the fact that $f e^{-R_{i}}$ is a finite measure. This concludes the proof of Theorem 2.17.

We finally obtain the Gallavotti-Cohen fluctuation theorem

Theorem 2.18 There is a neighborhood $O$ of the interval $\left[-\langle\sigma\rangle_{\nu},\langle\sigma\rangle_{\nu}\right]$ such that for $A \subset O$ the fluctuations of $\sigma_{i}$ in $A$ satisfy the large deviation principle with a large deviation functional $I(w)$ obeying

$$
I(w)-I(-w)=-w,
$$

i.e., the odd part of $I$ is linear with slope $-1 / 2$.

Proof: First we note that $e(\alpha)$ is a real analytic function since it is identified with an eigenvalue of a compact operator. A simple computation gives that

$$
\left.\frac{d}{d \alpha} e(\alpha)\right|_{\alpha=0}=\langle\sigma\rangle_{\nu} .
$$

The function $e(\alpha)$ is analytic and convex. By the result of [7] it is not identically zero, and so the symmetry the symmetry $e(\alpha)=e(1-\alpha)$ implies that the set of the values of $\frac{d}{d \alpha} e(\alpha)$ is a neighborhood of $\left[-\langle\sigma\rangle_{\nu},\langle\sigma\rangle_{\nu}\right]$.

The large deviation principle is a direct application of the Gärtner-Ellis theorem, 㕶, Theorem 2.3.6. The large deviation functional is given by the Legendre transform of $e(\alpha)$ and so we have

$$
\begin{aligned}
I(w) & =\sup _{\alpha}\{e(\alpha)-\alpha w\}=\sup _{\alpha}\{e(1-\alpha)-\alpha w\} \\
& =\sup _{\beta}\{e(\beta)-(1-\beta) w\}=I(-w)-w .
\end{aligned}
$$

\section{References}

[1] Balaji, S. and Meyn, S. P.: Multiplicative ergodicity and large deviations for an irreducible Markov chain. Stoch. Proc. Appl. 90 123-144 (2000). 
[2] Crooks, G.E.: Path-ensemble averages in systems driven far from equilibrium. Phys. Rev. E 61, 2361-2366 (2000)

[3] Deuschel, J.-D. and Stroock, D.W.: Large deviations. Pure and Applied Mathematics 137. Boston: Academic Press, 1989

[4] Dembo, A. and Zeitouni, O.: Large deviations techniques and applications. Applications of Mathematics 38. New-York: Springer-Verlag 1998

[5] Eckmann, J.-P. and Hairer, M.: Non-equilibrium statistical mechanics of strongly anharmonic chains of oscillators. Commun. Math. Phys. 212, 105$164(2000)$

[6] Eckmann, J.-P., Pillet C.-A., and Rey-Bellet, L.: Non-equilibrium statistical mechanics of anharmonic chains coupled to two heat baths at different temperatures. Commun. Math. Phys. 201, 657-697 (1999)

[7] Eckmann, J.-P., Pillet, C.-A., and Rey-Bellet, L.: Entropy production in nonlinear, thermally driven Hamiltonian systems. J. Stat. Phys. 95, 305-331 (1999)

[8] Evans, D.J., Cohen, E.G.D., and Morriss, G.P.: Probability of second law violation in shearing steady flows. Phys. Rev. Lett. 71, 2401-2404 (1993)

[9] Gallavotti, G. and Cohen E.G.D.: Dynamical ensembles in stationary states. J. Stat. Phys. 80, 931-970 (1995)

[10] Gentile, G.: Large deviation rule for Anosov flows. Forum Math. 10 89-118 (1998)

[11] Greiner, G: Spectral theory of positive semigroups on Banach lattices. In One-parameter semigroups of positive operators Lecture Notes in Mathematics 1184, Ed. R. Nagel, Berlin: Springer, 1986, pp 292-332

[12] Hörmander, L.: The Analysis of linear partial differential operators. Vol III, Berlin: Springer, 1985

[13] Jaksic V. and Pillet C.-A.: On entropy production in quantum statistical mechanics. Commun. Math. Phys. 217, 285-293 (2001)

[14] Jaksic V.and Pillet C-A.: Non-equilibrium steady states of finite quantum systems coupled to thermal reservoirs. Preprint (2001)

[15] Jarzynski, C.: Hamiltonian derivation of a detailed fluctuation theorem. J. Statist. Phys. 98, 77-102 (2000)

[16] Has'minskii, R.Z.: Stochastic stability of differential equations. Alphen aan den Rijn - Germantown: Sijthoff and Noordhoff, 1980 
[17] Kurchan, J: Fluctuation theorem for stochastic dynamics. J. Phys.A 31, 3719-3729 (1998)

[18] Maes, C. The fluctuation theorem as a Gibbs property. J. Stat. Phys. 95 367-392 (1999)

[19] Maes, C. Statistical mechanics of entropy production: Gibbsian hypothesis and local fluctuations. Preprint (2001)

[20] Maes, C., Redig, F., and Verschuere, M. No current without heat Preprint (2000)

[21] Meyn, S.P. and Tweedie, R.L.: Markov Chains and Stochastic Stability. Communication and Control Engineering Series, London: Springer-Verlag London, 1993

[22] Lebowitz, J.L. and Spohn, H.: A Gallavotti-Cohen-type symmetry in the large deviation functional for stochastic dynamics. J. Stat. Phys. 95, 333-365 (1999)

[23] Norriss, J.: Simplified Malliavin Calculus. In Séminaire de probabilités XX, Lectures Note in Math. 1204, 0 Berlin: Springer, 1986, pp. 101-130

[24] Pillet, C.-A.: Entropy production in classical and quantum systems. Markov Proc. Relat. Fields 7, 145-157, (2001).

[25] Rey-Bellet, L. and Thomas, L.E.: Asymptotic behavior of thermal nonequilibrium steady states for a driven chain of anharmonic oscillators. Commun. Math. Phys. 215, 1-24 (2000)

[26] Rey-Bellet, L. and Thomas, L.E.: Exponential convergence to non-equilibrium stationary states in classical statistical mechanics To appear in Commun. Math. Phys.

[27] Ruelle, D.: Entropy production in quantum spin systems. Preprint (2000)

[28] Stroock, D.W. and Varadhan, S.R.S.: On the support of diffusion processes with applications to the strong maximum principle. In Proc. 6-th Berkeley Symp. Math. Stat. Prob., Vol III, Berkeley: Univ. California Press, 1972, pp. $361-368$

[29] Wu, L.: Uniformly integrable operators and large deviations for Markov processes. J. Funct. Anal. 172, 301-376 (2000) 